The increase in the number of health facilities as well as the number of cases could reflect in part the positive impact of actions implemented by the state programme.

\section{P3.298 TRENDS AND ASSOCIATIONS OF TRICHOMONAS VAGINALIS INFECTION IN MEN AND WOMEN WITH GENITAL DISCHARGE SYNDROMES IN JOHANNESBURG, SOUTH AFRICA}

doi:10.1136/sextrans-2013-051184.0753

'D V Maseko, ${ }^{2} \mathrm{~K}$ Marsh, ${ }^{1} \mathrm{~F}$ Radebe, ${ }^{2} \mathrm{G}$ Hughes, ${ }^{1,3,4} \mathrm{D}$ A Lewis. ${ }^{1}$ Centre for HIV and STIs, National Institute for Communicable Diseases, National Health Laboratory Service, Johannesburg, South Africa; ${ }^{2}$ IIV and STI Department, Health Protection Services Colindale, London, UK; ${ }^{3}$ Department of Internal Medicine, Faculty of Health Sciences, University of the Witwatersrand, Johannesburg, South Africa; ${ }^{4}$ Division of Medical Microbiology, University of Cape Town, Cape Town, South Africa

Objectives To better understand the epidemiology of Trichomonas vaginalis infection, we investigated the association between $T$. vaginalis and demographic, clinical, microbiological and behavioural characteristics of patients presenting with genital discharges to a primary healthcare clinic in Johannesburg, South Africa.

Methods During six annual surveys (2007-2012), 1,218 male urethral discharge syndrome (MUDS) and 1,232 vaginal discharge syndrome (VDS) cases were consecutively recruited. Diagnostic methods included nucleic acid amplification (Neisseria gonorrhoeae, Chlamydia trachomatis, T. vaginalis, Mycoplasma genitalium), microscopy (bacterial vaginosis, Candida) and serology (Treponema pallidum, HSV-2, HIV). Chi-squared tests and logistic regression analyses were used to identify predictors of $T$. vaginalis infection.

Results T. vaginalis prevalence decreased from 2007 to 2012 (men, $13.4 \%$ to $4.8 \%$, p < 0.001 ; women, $33.8 \%$ to $23.1 \%$, p < 0.001 ). Overall, $74(6.1 \%)$ men and 291 (23.6\%) women were T. vaginalis positive, with the highest prevalence in those aged $\geq 40$ years (men, $13.6 \%$; women, $30.9 \%$ ). T. vaginalis infection occurred more often in pregnant women (adjusted odds ratio, aOR, 2.67; 95\% confidence intervals, CI, 1.29-5.54) and women with serological evidence of $T$. pallidum (aOR, 1.63; 95\% CI 1.08-2.45) or HSV-2 infections (aOR $1.75 ; 95 \%$ CI 1.16-2.64). T vaginalis infection occurred less often in men with co-existent gonorrhoea (aOR $0.35,95 \% \mathrm{CI}, 0.21-0.57$ ) and in women with either BV (aOR 0.60, 95\% CI 0.44-0.82) or Candida morphotypes (OR 0.61, 95\% CI 0.43-0.86).

Conclusions Although the prevalence of $T$. vaginalis infection decreased over time, it remains an important cause of genital discharge in South Africa, particularly in older patients and pregnant women.

\section{P3.299 TETRACYCLINE RESISTANCE IN UREAPLASMA SPECIES ISOLATED FROM WOMEN PRESENTING FOR TERMINATION OF PREGNANCY IN PRETORIA, SOUTH AFRICA}

doi:10.1136/sextrans-2013-051184.0754

M Le Roux, B E De Villiers, M R M Ditsele, S T Monokoane, L M Ngobeni. University of Limpopo (Medunsa Campus), Ga-Rankuwa, South Africa

Background The risks of untreated sexually transmitted infections in association with termination of pregnancy are known to increase the risk of post-termination complications. Local studies have shown a high genital tract carriage of mycoplasmas. Tetracyclines are widely used as first line agents against when ureaplasmal infection is detected However, there is limited data on the susceptibility profiles and mechanisms of resistance amongst Ureaplasma strains circulating in the Pretoria community. This study was undertaken to determine antimicrobial resistance to tetracycline among Ureaplasma species isolated from women presenting for termination of pregnancy at a tertiary referral hospital in South Africa.
Methods Two vaginal swabs were collected from each of 100 women after written informed consent was obtained. The first swab was used for PCR detection of genital ureaplasmas and tetracycline- resistance genes. The second swab was used for culture (Mycoplasma Duo kit) and phenotypic antimicrobial susceptibility testing (SIR Mycoplasma kit).

Results Ureaplasma species were isolated from 46 women (46\%). Forty-two women were infected with $U$. urealyticum, 1 with $U$. parvum and 3 were dually infected. Susceptibility profiles were obtained for 41 isolates. Fifteen $(36.6 \%)$ demonstrated resistance to tetracycline. Fourteen (34.1\%) were also resistant to doxycycline with 1 isolate showing intermediate resistance. All 41 strains contained the tet $M$ gene, 39 contained the $1.7 \mathrm{~kb}$ fragment of the tetracycline resistance gene and 36 strains contained the int-Tn gene.

Conclusion Genital ureaplasmas were isolated from nearly half the study population of women presenting for TOP. The predominant species identified was $U$ urealyticum. Tetracycline and doxycycline resistance was detected nearly a third of the isolates and this has implications for management of patients to prevent post-partum complications. The study will be expanded and communicated to the National Department of Health as this will impact future strategies of intervention in this country.

\section{P3.300 ASSESMENT OF SEXUAL BEHAVIOUR OF ALBANIAN HIGH SCHOOL STUDENTS}

doi:10.1136/sextrans-2013-051184.0755

'A Harxhi, ${ }^{2} \mathrm{~L}$ Merkuri, ${ }^{3} \mathrm{E}$ Agolli, ${ }^{4} \mathrm{~A}$ Agolli, ${ }^{5} \mathrm{~A}$ Subashi. ${ }^{1}$ University Hospital Center of Tirana, Tirana, Albania; ${ }^{2}$ Institute of Public Health, Tirana, Albania; ${ }^{3}$ UNFPA office, Tirana, Albania; ${ }^{4}$ Faculty of Social Science, Tirana, Albania; ${ }^{5}$ University Vitrina, Tirana, Albania

Background The aim of this study is to assess the sexual behaviour of high school youngsters in Albania in order to design better behaviour change interventions targeting this group of young people.

Methods This is a quantitative behavioural survey targeting young pupil attending high schools carried out in all regions in Albania. A representative sample of 2172 pupils of age group 15-19 years old was included in the study. A stratified random sampling technique was used to obtain this sample. A self administered structured questionnaire was used to collect data. Data analysis was made using SPSS version 12.

Results Almost $30 \%$ of participants of the study reported having had sexual intercourse, with a median age at first intercourse of 15 years. Only $23 \%$ of those experiencing sexual intercourse have ever used a condom. The reason for using it is to prevent an unwanted pregnancy and not to prevent sexually transmitted infections. $90 \%$ of the young people identified pharmacy stores as the only place to find condoms, while the family planning centres are not considered at all. Majority of young people did not discuss issues regarding condom use with their sexual partners.

Conclusion Although sexual education is part of the school curricula since few years, further strategies and interventions are needed to address issues regarding delaying first sexual intercourse and increasing condom use among high school young people in Albania.

\section{P3.301 MALE CLIENTS OF SEX WORKERS IN THE UNITED STATES: CORRELATES WITH STI/HIV RISK BEHAVIORS AND URBANIZATION LEVEL}

doi:10.1136/sextrans-2013-051184.0756

M A Villarroel. Johns Hopkins Bloomberg School of Public Health, Baltimore, MD, United States

Background Men who have purchased sex from sex workers ("clients") have been associated with an increased risk of acquiring STIs including HIV and in influencing the spread of infection in the 
population. To date, only a few reports on behavioural risk correlates and infection history of male clients have been documented in the United States, mainly drawn from populations in central cities and at high-risk for HIV. This study examines STIs/HIV behavioural risks and screening history correlates of male clients in the general population and whether the prevalence of male clients differs by urbanisation level.

Methods Data from the 1999-2000 National STD and Behavioral Measurement Study, a cross-sectional telephone-based probability sample of the non-institutionalised population aged 18 to 45 years old, was linked to the National Center Health Statistics Urban Rural Classification Scheme for Counties. The analysis is restricted to men aged 26 to 45 years old $(\mathrm{N}=469)$. F-statistic that accounted for survey design effects and Poisson regression for weighted data were used.

Results The overall prevalence of clients was 14.5 (95\% CI 11.518.1) and did not statistically differ between men residing in central cities of large metropolitan areas (MA) and men residing in large metropolitan suburbs, MAs of less than one million people, or nonmetropolitan subdivisions $(\mathrm{P}=0.5655)$. Compared to non-clients, clients had a significantly higher prevalence of an array of other high-risk behaviours in their lifetime and more recently including history of same-sex partners (Adjusted prevalence ratio (Adj. $P R)=2.5$, 95\% CI, 1.5-4.1), sold sex (Adj.PR = 3.6, 95\% CI, 2.2-6.1), multiple partners in the past year (Adj.PR $=2.6,95 \% \mathrm{CI}, 1.6-4.0$ ), as well as a history of STIs (Adj.PR $=2.595 \%$ CI 1.5-4.4).

Conclusion Male clients are ubiquitous. The risk-taking behaviour of clients in the general population is important to develop more comprehensive prevention measures for STIs/HIV.

\section{P3.302 SEXUAL HEALTH EXPERIENCE AND KNOWLEDGE OF HUMAN PAPILLOMAVIRUS (HPV) AND CHLAMYDIA IN YOUNG WOMEN RECRUITED VIA SOCIAL NETWORKING SITES}

doi:10.1136/sextrans-2013-051184.0757

'S M Garland, ${ }^{2} \mathrm{~B}$ Gunasekaran, ${ }^{3} \mathrm{~N}$ Ahmed, ${ }^{4} \mathrm{Y}$ Fenner, ${ }^{1} Y$ Jayasinghe, ${ }^{5} \mathrm{E}$ Moore, ${ }^{4} \mathrm{~A}$ Fletcher, 'S Tabrizi, ${ }^{6} \mathrm{~J}$ Wark. 'Dept of Microbiology \& Infectious Diseases The Royal Womens Hospital, Dept of Obstetrics \& Gynaecology, University of Melbourne \& Dept of Microbiology, The Royal Childrens Hospital \& Murdoch Childrens Research Institute, Parkville, Australia; ${ }^{2}$ Dept of Microbiology \& Infectious Diseases The Royal Womens Hospital, Dept of Microbiology The Royal Childrens Hospital \& Murdoch Childrens Researh Institute, Parkville, Australia; ${ }^{3}$ Dept of Microbiology \& Infectious Diseases The Royal Womens Hospital, Dept of Obstetrics \& Gynaecology, University of Melbourne, Parkville, Australia; ${ }^{4}$ Dept of Microbiology \& Infectious Diseases The Royal Womens Hospital, Dept of Microbiology, The Royal Childrens Hospital \& Murdoch Childrens Research Institute, Parkville, Australia; ${ }^{5}$ Dept of Microbiology \& Infectious Diseases The Royal Womens Hospital, Dept of Microbiology, The Royal Childrens Hospital \& Murdoch Childrens Research Institute, Parkville, Australia; ${ }^{6}$ Department of Medicine, Royal Melbourne Hospital, Department of Medicine, University of Melbourne, Parkville, Australia

Background Monitoring sexual health of young Australian women is a major research priority since introduction of the federally funded HPV vaccination programme, plus screening programmes for chlamydia. Social networking sites (SNS), commonly used by young people, present an opportunity for innovative recruitment modalities. We assessed young women's knowledge and experience of chlamydia, HPV, HPV vaccines and cervical cytology (Pap smears) utilising Facebook

Method This was part of a feasibility study assessing use of Facebook to recruit subjects for a larger prospective health study, the Young Female Health Initiative (YFHI). Women 16 to 25 years, from Victoria, Australia were eligible to participate. An advertisement was placed on Facebook for 6 months and visible to a random sample of eligible women. Women clicking on the advertisement were redirected to our website, then contacted and asked to complete a survey at the YFHI study centre, or the questionnaire online. The survey contained demographic questions, plus sexual health questions.

Results We enrolled 426 respondents, of whom 278 completed the survey $(50 \%$ study centre, $50 \%$ online). Respondents' socioeconomic and geographical distribution (urban, regional, rural) were representative of the target population; those $>18$ years were more likely to enrol than $16-17$ year olds ( $p<0.05$ ). Overall, $76 \%$ had been sexually active, median age of coitarche was 16.9 years [CI 16.6-17.2], 63\% had heard of HPV: of these, 73\% knew HPV is sexually acquired and $94 \%$ that it causes cancer. $78 \%$ had heard of chlamydia: those who were sexually active were more likely to know of chlamydia than were virgins $(p<0.01)$, while $63 \%$ knew it could cause chronic pelvic pain, and $86 \%$ that it could cause infertility. This recruitment method also was cost-effective (\$USD 20 per compliant participant).

Conclusions SNS is an effective recruitment strategy to engage young women in sexual health research

\section{P3.303 HIV PREVALENCE AND ASSOCIATED RISK FACTORS AMONG UNIVERSITY STUDENTS IN EASTERN ETHIOPIA, 2009}

doi:10.1136/sextrans-2013-051184.0758

\section{B Mengistu. NASTAD, Addis Abeba, Ethiopia}

Background The students in University enjoy the freedom of living outside the guidance of their parents; if this is not managed properly it increases the likelihood to have of risky sexual behaviour and HIV infection. It is imperative to monitor HIV among young people in universities for proper and timely action.

Objective This research objective was to determine the prevalence of HIV infection among Dire Dawa university students and identify risk factors for HIV sero-positivity.

Method A cross sectional study with internal comparison was conducted among students of Dire Dawa. A self administrated questionnaire was used to gather relevant information and blood test was conducted for HIV antibody. The two information were linked anonymously. Qualitative data was collected using Focus group discussion.

Result The prevalence of HIV was $2.5 \%$ (95\% CI 1.5, 3.5) (2.3\% for female and $2.7 \%$ for male) with no significant difference between male and female. The students having sexual affair with same sex or both sexes were significantly 10.45 times $(95 \%$ CI $1.93,56.41)$ to be exposed to HIV than those having sexual affairs with only opposite sex. Having non-regular partner is significantly associated with HIV positivity among sexually active $(\mathrm{OR}=6.35$ and $95 \%$ CI $2.2,18.3)$. Drinking alcohol and chewing chat was a risk factor for commencing sexual intercourse among male with $(\mathrm{OR}=2.73$ and $95 \% \mathrm{CI}$ $1.72,4.33)$ and $(\mathrm{OR}=2.02$ and $95 \%$ CI $1.19,3.42)$ respectively.

Conclusion The students in the university are at risk of HIV infection, it is necessary to target them earlier in the high school and later when joining college by building their knowledge and skill to avoid risky sexual behaviour.

\section{P3.304 WITHDRAWN BY AUTHOR}

\section{P3.305 VULNERABILITY OF SEX WORKERS (SWS) AND THEIR PARTICULAR NEEDS FOR HIV/STI PREVENTION, DIAGNOSIS, AND TREATMENT: BORDERNETWORK RESEARCH FINDINGS AND RECOMMENDATIONS FOR COMPREHENSIVE SEXUAL HEALTH RESPONSE}

doi:10.1136/sextrans-2013-051184.0759

'E Steffan, ${ }^{1} \mathrm{~T}$ Arsova Netzelmann, ${ }^{2} \mathrm{~L}$ Lõhmus, ${ }^{3} \mathrm{~J}$ Kalikov, ${ }^{4} \mathrm{~A}$ Karnite, ${ }^{5} \mathrm{~A}$ Leffers, ${ }^{6} \mathrm{~B}$ Kucharova, ${ }^{7} \mathrm{R}$ Dimitrova, ${ }^{8} \mathrm{C}$ Fierbinteanu. ${ }^{1} \mathrm{SPI}$ Forschung gGmbH, Berlin, Germany; ${ }^{2}$ National Institute for Health Development, Tallinn, Estonia; ${ }^{3} \mathrm{AIDS}$ Information and 